\title{
Impact of Acute Lumbar Disk Herniation on Sexual Function in Male Patients
}

\author{
Keerthivasan Panneerselvam, Rishi Mugesh Kanna, Ajoy Prasad Shetty, Shanmuganathan Rajasekaran \\ Department of Orthopaedics and Spine Surgery, Ganga Hospital, Coimbatore, India
}

\section{Study Design: Prospective observational study.}

Purpose: In this study, we determined the effects of lumbar disk herniation (LDH) on male sexual activity and whether surgical intervention improved any related sexual dysfunction.

Overview of Literature: The impact of surgery on sexual activity has been extensively studied in arthroplasty, uro-gynecological surgery, and stroke, but there are relatively few studies on spine surgery, and none involve an Asian population to the best of our knowledge.

Methods: We evaluated sexually active male patients ( $\mathrm{n}=22,40.8 \pm 6.8$ years) admitted for microdiscectomy with a questionnaire for assessing sexual function before and 8 weeks after surgery. The questionnaire included the Oswestry Disability Index, Hospital Anxiety Depression Score, and Brief Sexual Function Inventory (BSFI), as well as questions about perceived sexual dysfunction (frequency, performance, satisfaction).

Results: The average preoperative Visual Analog Scale (VAS) score was 4.36 \pm 2.59 ( $n=18)$ for low back pain (LBP) and $6.81 \pm 2.1$ ( $n=22$ ) for leg pain. The mean preoperative BSFI score was $27.8 \pm 11.2$. Among the five BSFI components, sexual drive was reduced in $63.0 \%$ of patients, while erection and ejaculation were affected in $40.9 \%$ and $31.8 \%$, respectively. The VAS score for LBP had a negative correlation with the preoperative BSFI score $(p<0.03)$. After LDH onset, $54.5 \%$ of patients noted a decrease in frequency, and $77.2 \%$ described a decrease in desire and satisfaction. At 8 weeks after surgery, the mean BSFI score significantly improved to 33.23 ( $p=0.002$ ). Sexual drive was normal in $77.7 \%$ of patients, and erection and ejaculation were normal in $77.7 \%$ and $91.0 \%$, respectively. Overall, $59.1 \%$ had resumed sexual intercourse within 6 weeks of surgery.

Conclusions: LDH resulted in sexual dysfunction in up to $77 \%$ of patients, which significantly improved after surgery. By 6 weeks, the majority had resumed sexual activity without undue discomfort. Therefore, this study supports counseling for patients with LDH about sexual function.

Keywords: Lumbar disc; Sexual dysfunction; Back pain; Male; Counseling

\section{Introduction}

Lumbar disk herniation (LDH), which presents with severe lower back pain (LBP) with radiation to the leg, can affect an individual's normal functions [1]. Among patients with LBP, up to $72 \%$ have reported varying degrees of disturbance in their sexual activity [2]. Sexual dysfunction in people with lumbar disc diseases can be

Received Mar 25, 2021; Revised Jun 24, 2021; Accepted Jul 27, 2021

Corresponding author: Rishi Mugesh Kanna

Department of Orthopaedics and Spine Surgery, Ganga Hospital, Coimbatore, Tamil Nadu, India

Tel: +91-9486600798, Fax: +91-0422-4364449, E-mail: rishiortho@gmail.com 
caused by actual pain or fear of aggravating pain, physical restriction, depression associated with chronic pain, or adverse medication effects [3]. The mental, physical, and emotional status of humans have strong associations with their sexual health; thus, sexual dysfunction can result in lowered self-esteem and hinder personal and social wellbeing [4].

Patients seeking surgical consultation for severe radiculopathy due to LDH inquire about physical activity modification, ergonomics, and rehabilitation but rarely about the effects of sexual activity. Sociocultural taboos and perceived stigma around sexuality can inhibit patients from discussing their sexual health, and most patients deliberately avoid talking about it due to modesty or fearing embarrassment [5]. Inadequate knowledge can further worsen the issue by means of overreaction (i.e., unwanted sexual abstinence) or insufficient measures (i.e., avoiding unusual postures or vigorous activity).

The highest prevalence of $\mathrm{LDH}$ is among patients aged $30-50$ years, which is also the period of peak sexual activity $[6,7]$. Studies have shown that lumbar flexion increases intradiscal pressure and men are mostly in a lumbar-flexed position during coitus [8]. Hence, the presence of LDH can affect sexual function in the following ways: (1) axial and radicular pain can be aggravated by sexual activity; (2) the presence of severe pain can decrease sexual performance; and (3) compression of the cauda equina nerve roots can result in altered sensation in the perineal areas, potentially affecting sexual performance and satisfaction. Therefore, it is essential to study the physiological and psychological effects of LBP and sciatica associated with LDH on sexual function. The impact of surgery on sexual activity has been extensively studied in arthroplasty, uro-gynecological surgery, and stroke [9-11], but studies on spine surgery are relatively limited, and none involve an Indian population to the best of our knowledge.

Spine surgeons should also have adequate knowledge about the effects of LDH on patients' sexual function and whether surgery positively or negatively affects sexual activity. Sexual desire, erection, ejaculation, and satisfaction are independent components of sexual function, and the extent of affliction in each by LDH has not been studied before. Hence, for proper preoperative counseling and to guide patients in performing postoperative activity with a certain level of confidence, surgeons must have knowledge about the effects of LDH and spine surgery on sexual activity. Therefore, in this study, we determined the effects of LDH on sexual activity and whether surgical intervention can improve any related sexual dysfunction.

\section{Materials and Methods}

We conducted this prospective, observational study in the Department of Spine Surgery of Ganga Medical Centre and Hospitals, Coimbatore, India from November 2018 to April 2019 after approval from the Institutional Review Board (IRB approval no., GMCHIRB/2019/03/05). We obtained informed written consent from all participants. We counseled all consecutive male patients aged $>18$ years with LBP and radicular leg pain with radiological evidence of LDH in magnetic resonance imaging and planned for microlumbar discectomy about the study, including those who were willing to participate with an informed consent. We excluded female patients, male patients $<18$ and $>60$ years of age, patients with significant motor power deficits or cauda equina syndrome, and those who were apprehensive about participating.

Before data collection, we clearly explained to the participants about the confidentiality of their identity and data and how we would provide proper privacy while they answered the questionnaire. The investigator established a good rapport with the participants and answered all of their queries, taking care to avoid any leading questions.

We recorded patients' preoperative demographic data, such as age, marital status, years of marriage, spouse's age, number of children, and mean symptom duration. Clinical data included pain type and duration, Visual Analog Scale (VAS) scores for LBP and leg pain, factors aggravating pain, comorbidities, and current medications. Questions regarding sexual function included usual weekly frequency of sexual intercourse, change in frequency after the onset of LDH pain, change in sexual desire and satisfaction after pain onset, and any specific sexual problems (e.g., decreased desire, premature or delayed ejaculation, erectile dysfunction) and their duration.

Since guidelines that discuss resuming sexual function after lumbar microsurgery are unavailable, we did not provide specific instructions to the patients about this issue, advising them to resume sexual function on the basis of their comfort levels. Hence, during the postoperative follow-up visit at 8 weeks, we sought the following details: When was first intercourse after surgery? Was there a change in sexual desire or satisfaction after surgery? If specific sexual problems were present before surgery, were 
they improved, the same, or worse after surgery?

We recorded scores on the Oswestry Disability Index (ODI) [12] and Brief Sexual Function Inventory (BSFI) for men in the preoperative period (considering the past 1 month before surgery) and at week 8 in the postoperative period [13] (Appendix 1). The BSFI, a discrete screening tool for sexual function, assesses sexual drive, erection, ejaculation, sexual problem perception, and overall satisfaction in male patients. Among these five components, each had a maximum score of $9,12,8,12$, and 4 , respectively, with a maximum total score of 45 . For analysis, we considered that a score of $<50 \%$ in each component showed impairment. We performed psychological screening for anxiety and depression using the Hospital Anxiety Depression Scale (HADS) in the preoperative period [14] (Appendix 2).

We analyzed the data using IBM SPSS software ver. 20.0 (IBM Corp., Armonk, NY, USA). We calculated descriptive statistics for the background variables. We used Pearson's correlation coefficient to determine the correlation between two continuous variables. We tested the association between categorical variables using the chisquare test. We performed the Mann-Whitney $U$-test and Wilcoxon signed-rank test for the quantitative variables, as these data do not follow normal distribution. We considered $p<0.05$ as statistically significant.

\section{Results}

\section{Patient demographics}

This study included 22 male patients with an average age of $40.78 \pm 6.87$ years. All patients were married for a mean $13.77 \pm 8.11$ years; their spouses' average age was $36.77 \pm 6.73$ years. Overall, $13.6 \%$ of patients were uneducated, while $45.3 \%$ had school-level education, and $40.7 \%$ were college graduates (Table 1). Four patients were manual laborers, nine were skilled workers, eight were professionals, and one was a businessman. All 22 patients had radiating leg pain, with 18 patients also having LBP. The average duration of symptoms was $6.88 \pm 3.27$ months. The level of pathology was L4-5 in 12 patients, L5-S1 in eight, and L3-4 in two.

\section{Patient outcomes scores}

The average preoperative VAS score was $4.36 \pm 2.59$ for
Table 1. Patient demographics

\begin{tabular}{|cc|}
\hline Characteristic & Value \\
\hline Age (yr) & $40.78 \pm 6.87$ \\
\hline Age of spouse (yr) & $36.77 \pm 6.73$ \\
\hline Years of married life (yr) & $13.79 \pm 8.11$ \\
\hline Educational status & \\
\hline Uneducated & 2 \\
\hline School & 11 \\
\hline Diploma & 1 \\
\hline Graduate & 8 \\
\hline Occupation & \\
\hline Unemployed & 0 \\
\hline Manual labour & 4 \\
\hline Skilled labour & 9 \\
\hline Professional & 8 \\
\hline Business & 1 \\
\hline Comorbidities & 2 \\
\hline Diabetes mellitus & 1 \\
\hline Hypertension & 1 \\
\hline Mitral regurgitation & \\
\hline Addictive habits & \\
\hline Smoking & \\
\hline Smoking \& alcohol & \\
\hline & \\
\hline
\end{tabular}

Values are presented as mean \pm standard deviation or number.

Table 2. Comparison between preoperative and postoperative functional scores

\begin{tabular}{|c|c|c|c|}
\hline Variable & $\begin{array}{l}\text { Before } \\
\text { surgery }\end{array}$ & $\begin{array}{l}\text { After surgery at } 8 \\
\text { weeks }\end{array}$ & $p$-value \\
\hline VAS-back pain & $4.36 \pm 2.59$ & $3.12 \pm 2.47$ & 0.11 \\
\hline VAS-leg pain & $6.81 \pm 2.10$ & $2.37 \pm 1.90$ & $<0.0001$ \\
\hline ODI & $29.5 \pm 9.48$ & $11.0 \pm 6.47$ & $<0.0001$ \\
\hline BSFI & $27.86 \pm 11.17$ & $33.23 \pm 9.46$ & 0.002 \\
\hline \multicolumn{4}{|l|}{ Reduced performance (\%) } \\
\hline Sexual drive & 63.0 & 22.3 & 0.006 \\
\hline Erection & 40.9 & 22.3 & 0.19 \\
\hline Ejaculation & 31.8 & 9.0 & 0.06 \\
\hline Problem assessment & 36.3 & 9.0 & 0.03 \\
\hline Satisfaction & 45.4 & 36.3 & 0.76 \\
\hline
\end{tabular}


LBP and $6.81 \pm 2.10$ for leg pain. The mean ODI score was 29.50 9 9.48. In the HADS scoring for anxiety, nine patients were in the normal range, six were borderline, and seven were in the anxiety range. For depression, seven patients were normal, seven were borderline, and eight in the depressed range.

In the preoperative period, the mean BSFI score was 27.86 \pm 11.17 . Among the five BSFI components, sexual desire was reduced in $63.0 \%$ of the patients, while erection and ejaculation were reduced in $40.9 \%$ and $31.8 \%$, respectively. For satisfaction with sexual activity, $45.4 \%$ observed it to be low (Table 2). Patient age, years of marriage, and comorbidity affected the preoperative BSFI score, whereas addictive habits had no influence (Table 3). Univariate analysis showed a significant decrease in the BSFI problem assessment component in patients $>40$ years of age, in overall satisfaction in patients married for $>10$ years, and in ejaculation, problem assessment, and total scores in patients with comorbidities.

The LBP VAS scores had a negative correlation with the preoperative BSFI scores $(p<0.03)$, whereas those for leg pain had no significant correlation. Pain duration had no significant bearing on BSFI score. There was an inverse correlation between ODI and preoperative BSFI scores, as well as postoperative BSFI scores $(p<0.01)$. We noted a statistically significant negative correlation between the HADS anxiety scores and the preoperative BSFI scores $(p<0.01)$, as well as the postoperative BSFI scores $(p<0.02)$; the HADS depression scores had no statistical significance (Table 4).

\section{Frequency}

The frequency of sexual intercourse in the preoperative period (the 3 months before surgery) was mentioned as $2-3 \times$ per week by nine patients, once per week by nine patients, and rarer than once per week by four patients. After LDH development, 54.4\% of the patients reported a decrease in the frequency of sexual intercourse, and $77.3 \%$ described a decrease in desire or satisfaction. The change in frequency and desire had a statistically significant correlation with the preoperative BSFI scores $(p<0.001)$.

\section{Patient postoperative scores}

At 8 weeks after surgery, the mean ODI score was 11.0 \pm 6.5 . The VAS score for LBP decreased to $3.12 \pm 2.47$

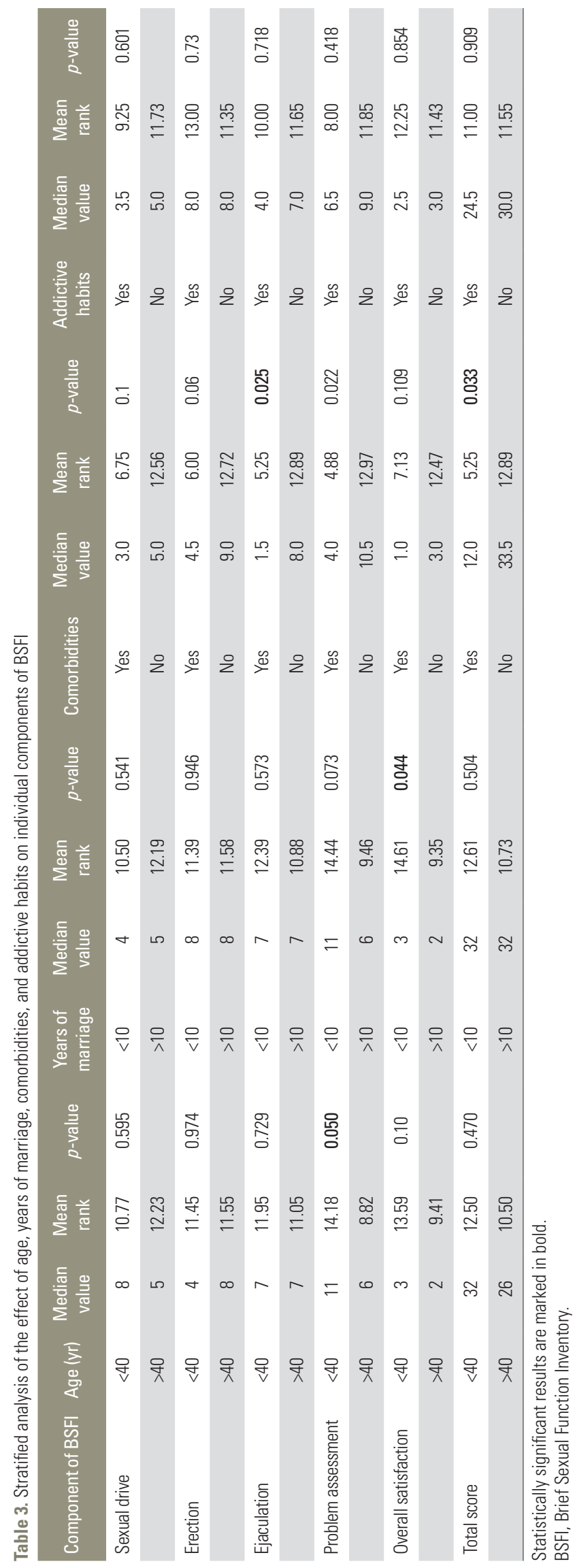


Table 4. Correlation between various clinical parameters and BSFI

\begin{tabular}{llccc} 
Parameter 1 & Parameter 2 & Pearson correlation & Significance (2-tailed) & Significance \\
\hline Duration of pain & BSFI-Preop & -0.163 & 0.470 & Not significant \\
Duration of pain & BSFI-Postop & -0.011 & 0.962 & Not significant \\
HADS-anxiety & BSFI-Preop & -0.514 & 0.014 & Significant \\
HADS-anxiety & BSFI-Postop & -0.479 & 0.024 & Significant \\
HADS-depression & BSFI-Preop & -0.241 & 0.279 & Not significant \\
HADS-depression & BSFI-Postop & -0.240 & 0.283 & Not significant \\
\hline ODI & BSFI-Preop & -0.596 & 0.003 & Significant \\
\hline BSF, Bit
\end{tabular}

BSFI, Brief Sexual Function Inventory; Preop, preoperative; Postop, postoperative; HADS, Hospital Anxiety Depression Scale; ODI, Oswestry Disability Index.

and that for leg pain to $2.37 \pm 1.9$. The postoperative ODI and VAS for leg pain scores showed significant improvements ( $p<0.05$ ); the VAS score for LBP did not (Table 2 ).

The mean postoperative BSFI score showed a significant improvement (33.23 $\pm 9.46, p=0.002)$. Among the five subcomponents, sexual drive, ejaculation, and problem perception showed statistically significant improvements $(p<0.05)$ (Table 2). Erection and sexual satisfaction also showed postoperative improvements, although the $p$ value was $>0.05$. Of the 17 patients who mentioned a decrease in sexual desire or satisfaction, 15 (88.2\%) had subjective improvement in the postoperative period, while two had no difference. The majority of patients, $59.1 \%$, had resumed sexual intercourse within 6 weeks of surgery. The remaining patients mentioned the fear of eliciting pain as the reason for delaying sexual activity.

\section{Discussion}

We observed that lack of sexual desire leading to decreased frequency was common in up to $77 \%$ of patients with LDH. Patients with coexistent LBP had greater affliction than did those with isolated radiculopathy. Surgery significantly improved the pain with corresponding improvements in sexual function. Pain associated with LDH has moderate-to-severe detrimental effects on various aspects of life and sexual dysfunction due to physical constraint, emotional distress, and psychological factors [15-17]. The incidence of LDH is approximately 5-20 per 1,000 adults, involving more male patients in their third to fifth decades of life [18]. However, the afflicted patients rarely made any inquiries regarding sexual activity due to the taboos around the subject. Counseling about sexual dysfunction by the treating surgeon or physiotherapist is also deficient due to the lack of knowledge or specialized training and patient's lack of initiative [19].

Psychological factors can play a vital role in sexual performance [20]. Psychological assessment in our study indicated that $>60 \%$ of patients had anxiety (definite and borderline), which statistically correlated to preoperative sexual dysfunction. Akbas et al. [21] had similar findings in their study on 43 patients with LDH in which $59 \%$ had HADS scores indicating anxiety, and a significant statistical correlation was noted between anxiety and sexual dysfunction. Anxiety can be caused by pain, fear about the surgical intervention and its consequences, or misperceptions about the disease itself. Patients who are more anxious tend to avoid any activity they perceive as aggravating pain, which can explain the decreased desire and satisfaction with sexual activity. Furthermore, individuals with anxiety disorder are more prone to performance anxiety related to sex [20], which can lead to decreased desire and satisfaction.

Horst et al. [22] and Kwan et al. [23] have observed a correlation between depression and sexual dysfunction, and although there was a negative correlation between depression and BSFI score in our study, the statistical significance was weak. Depression initially affects the sexual drive alone, and other factors are involved only after a long duration of chronic illness. However, the average symptom duration in our study population was only 6 months, which can explain the lesser effects of depression on sexual function. Furthermore, the study population was relatively young, and as such, depression may be superseded by anxiety.

The BSFI assesses the sexual drive, erection, ejaculation, problem assessment, and overall satisfaction in male. The maximum BSFI score is 45 which indicate a perfect sexual 
function. In our study, the total preoperative BSFI score was 27.86, which was inversely related to the ODI score. ODI measures the extent of disability in a patient with spinal disorders and associated back or leg pain. The significant statistical correlation between the VAS scores for LBP and preoperative BSFI scores is understandable, because LBP can affect sexual activity directly and indirectly. While the preoperative BSFI scores indicated an overall decrease in sexual function, the five individual components also showed a decline. Sexual drive was low in most patients, $63 \%$, followed by difficulties with erection and ejaculation. Correspondingly, sexual satisfaction was low in $45 \%$ of the patients.

All patients had good functional outcomes after surgery as measured by the ODI, and of the 17 patients who mentioned a decrease in desire and satisfaction with sexual activity, 15 had improvement in both areas after the surgery. Kanayama et al. [24] have concluded in their study that surgical treatment improved the quality of sexual activities, and our study had a similar finding of statistically significant improvement in BSFI scores in the postoperative follow-up period as compared to those of the preoperative period. Sexual drive improved in $78.7 \%$ of the patients, while ejaculation and erection improved in $91.0 \%$ and $77.7 \%$, respectively.

Owing to the lack of existing guidelines, the patients were not advised about a specific waiting period before returning to sexual activity after surgery. The majority had resumed sexual activity within 6 weeks of surgery; the remainder cited a fear of eliciting pain as the reason for the delay. No patients reported any increase in pain after their first sexual intercourse after surgery. These findings underline the importance of proper counseling about sexual activity in the postoperative period to avoid unnecessary abstinence, which can lead to emotional distress and interpersonal relationship issues [25].

Considering the lack of data about the optimal time for resuming sexual activity after spine surgery [26], the advice provided by the spine surgeon or physiotherapist can depend on their own clinical experience and their postsurgical exercise guidelines. Early return to sexual activity can increase pain, which can result in anxiety and a consequent fear about sex, whereas an unnecessary delay in returning can lead to interpersonal distress. Therefore, it is prudent to develop an appropriate protocol for return to sexual activity after spine surgery.

Since sexual activity is considered a medium-level physical activity similar to brisk walking or bicycling, a 6 -week wait is considered reasonable before resuming sexual activity after spinal surgery [27]. In the absence of guidelines, most of our patients participated in sexual activity within 6 weeks after surgery, which we consider is an appropriate time period that can be discussed during patient counseling.

This study has several limitations. First, the follow-up period of 2 months was short, as participants might have further symptom improvement over time. Second, the number of participants was small.

\section{Conclusions}

$\mathrm{LDH}$ resulted in sexual dysfunction in $77 \%$ of the patients, which significantly improved after surgery. Patients with a combination of axial and radicular pain were more affected than were those with pure radicular pain, and anxiety rather than depression was more detrimental to patient sexual health. Proper counseling regarding postoperative sexual activity is crucial for patient optimal overall functional outcomes.

\section{Conflict of Interest}

No potential conflict of interest relevant to this article was reported.

\section{Acknowledgments}

The study was funded by the Ganga Orthopaedic Research and Education Foundation (GOREF).

\section{Author Contributions}

Conception and design: PK, RMK; Analysis and interpretation: PK, RMK; Writing the article: PK, RMK; Data collection: PK; Provision of materials, patients, or resources: RMK, APS, SR; Statistical expertise: PK, RMK; Obtaining funding, RMK, APS, SR; Literature search: PK; Administrative, technical, or logistic support: RMK, SR; Critical revision of the article: APS, SR; Final approval of the article: RMK, APS, SR. 


\section{References}

1. Deyo RA, Mirza SK. Clinical practice: herniated lumbar intervertebral disk. N Engl J Med 2016;374:176372 .

2. Nikoobakht M, Fraidouni N, Yaghoubidoust M, Burri A, Pakpour AH. Sexual function and associated factors in Iranian patients with chronic low back pain. Spinal Cord 2014;52:307-12.

3. Maigne JY, Chatellier G. Assessment of sexual activity in patients with back pain compared with patients with neck pain. Clin Orthop Relat Res 2001;(385):827.

4. Wagner G, Fugl-Meyer KS, Fugl-Meyer AR. Impact of erectile dysfunction on quality of life: patient and partner perspectives. Int J Impot Res 2000;12 Suppl 4:S144-6.

5. Latifnejad Roudsari R, Javadnoori M, Hasanpour M, Hazavehei SM, Taghipour A. Socio-cultural challenges to sexual health education for female adolescents in Iran. Iran J Reprod Med 2013;11:101-10.

6. Beutel ME, Stobel-Richter Y, Brahler E. Sexual desire and sexual activity of men and women across their lifespans: results from a representative German community survey. BJU Int 2008;101:76-82.

7. Flynn KE, Lin L, Bruner DW, et al. Sexual satisfaction and the importance of sexual health to quality of life throughout the life course of U.S. adults. J Sex Med 2016;13:1642-50.

8. Sidorkewicz N, McGill SM. Male spine motion during coitus: implications for the low back pain patient. Spine (Phila Pa 1976) 2014;39:1633-9.

9. Issa K, Pierce TP, Brothers A, Festa A, Scillia AJ, Mont MA. Sexual activity after total hip arthroplasty: a systematic review of the outcomes. J Arthroplasty 2017;32:336-40.

10. Walker LM, Wassersug RJ, Robinson JW. Psychosocial perspectives on sexual recovery after prostate cancer treatment. Nat Rev Urol 2015;12:167-76.

11. Boller F, Agrawal K, Romano A. Sexual function after strokes. Handb Clin Neurol 2015;130:289-95.

12. Fairbank JC, Pynsent PB. The Oswestry Disability Index. Spine (Phila Pa 1976) 2000;25:2940-52.

13. Mykletun A, Dahl AA, O’Leary MP, Fossa SD. Assessment of male sexual function by the Brief Sexual Function Inventory. BJU Int 2006;97:316-23.

14. Herrmann C. International experiences with the
Hospital Anxiety and Depression Scale: a review of validation data and clinical results. J Psychosom Res 1997;42:17-41.

15. Gruenwald I, Yehieli-Cohen R, Adler T, Haddad M, Eisenberg E. PS-08-006 Sexual dysfunction in patients with chronic pain. J Sex Med 2016;13:S112-3.

16. Berg S, Fritzell P, Tropp H. Sex life and sexual function in men and women before and after total disc replacement compared with posterior lumbar fusion. Spine J 2009;9:987-94.

17. Malik AT, Jain N, Kim J, Khan SN, Yu E. Sexual activity after spine surgery: a systematic review. Eur Spine J 2018;27:2395-426.

18. Long DM, BenDebba M, Torgerson WS, et al. Persistent back pain and sciatica in the United States: patient characteristics. J Spinal Disord 1996;9:40-58.

19. Zebala L, Cronen G, Buchowski J, et al. P63 Sexual relations after spine surgery: do patients have questions that are not being addressed? Spine J 2009;9(10 Suppl):148S.

20. Rajkumar RP, Kumaran AK. Depression and anxiety in men with sexual dysfunction: a retrospective study. Compr Psychiatry 2015;60:114-8.

21. Akbas NB, Dalbayrak S, Kulcu DG, Yilmaz M, Yilmaz T, Naderi S. Assessment of sexual dysfunction before and after surgery for lumbar disc herniation. J Neurosurg Spine 2010;13:581-6.

22. Horst PK, Khanna K, Racine L, et al. Sex life and impact of operative intervention on sex life-related pain in degenerative spinal conditions: an analysis of the SPORT study. Spine (Phila Pa 1976) 2016;41:1764-71.

23. Kwan KS, Roberts LJ, Swalm DM. Sexual dysfunction and chronic pain: the role of psychological variables and impact on quality of life. Eur J Pain 2005;9:643-52.

24. Kanayama M, Horio M, Umi Y, et al. How does surgery affect sexual desire and activities in patients with lumbar disc herniation? Spine (Phila Pa 1976) 2010;35:647-51.

25. Korse NS, Nicolai MP, Both S, Vleggeert-Lankamp CL, Elzevier HW. Discussing sexual health in spinal care. Eur Spine J 2016;25:766-73.

26. Thomas CM, Levene HB. Lack of current recommendations for resuming sexual activity following spinal surgery. Asian Spine J 2019;13:515-8.

27. Frappier J, Toupin I, Levy JJ, Aubertin-Leheudre M, Karelis AD. Energy expenditure during sexual activity in young healthy couples. PLoS One 2013;8:e79342. 
Appendix 1. A Brief Sexual Function Inventory

\section{Sexual drive}

Let's define sexual drive as a feeling that may include wanting to have a sexual experience (masturbation or intercourse) thinking about having sex or felling frustrated due to lack of sex.

1) During the past 30 days, on how many days have you felt sexual drive?

\begin{tabular}{lcccc} 
No days everyday & Only a few days & Some days & Most days & Almost \\
1 & 2 & 3 & 4 & 5 \\
\hline 2) During the past 30 days, how would you rate your level of sexual drive? & & & Medium high & High \\
Not at all & Low & Medium & 3 & 4 \\
0 & 1 & 2 & 3 \\
\hline
\end{tabular}

Erections

3) Over the past 30 days, how often have you had partial or full sexual erections when you were sexually stimulated in any way?

\begin{tabular}{lcccc} 
Not at all & A few times & Fairly often & Usually & Always \\
0 & 1 & 2 & 3 & 4 \\
\hline
\end{tabular}

4) Over the past 30 days, how often have you had erections; how often were they firm enough to have sexual intercourse?

\begin{tabular}{lcccc} 
Not at all & A few times & Fairly often & Usually & Always \\
0 & 1 & 2 & 3 & 4 \\
\hline
\end{tabular}

5) How much difficulty did you have getting an erection during the last 30 days?

\begin{tabular}{ccccc} 
No erections at all & A lot & Some & Little & No difficulty \\
0 & 1 & 2 & 3 & 4 \\
\hline
\end{tabular}

\section{Ejaculation}

6) Over the past 30 days how much difficulty have you had in ejaculating when you have been sexually stimulated?

\begin{tabular}{lcccc} 
Have not had sexual stimulation in the past month & A lot & Some & Little & No difficulty \\
\hline 0 & 1 & 2 & 3 & 4 \\
\hline
\end{tabular}

7) In the past 30 days, how much did you consider the amount of semen you ejaculate?

\begin{tabular}{lcccc} 
Did not climax & Big problem & Medium problem & Small problem & No problem \\
0 & 1 & 2 & 3 & 4 \\
\hline
\end{tabular}

Problem assessment

8) In the past 30 days, to what extent have you considered a lack of sex drive to be a problem?

\begin{tabular}{lcccc} 
Big problem & Medium problem & Small problem & Very small problem & No problem \\
0 & 1 & 2 & 3 & 4 \\
\hline
\end{tabular}

9) In the past 30 days, to what extent have you considered your ability to get and keep an erection a problem?

\begin{tabular}{lcccc} 
Big problem & Medium problem & Small problem & Very small problem & No problem \\
0 & 1 & 2 & 3 & 4 \\
\hline
\end{tabular}

10) In the past 30 days, to what extent have you considered your ejaculation to be a problem?

\begin{tabular}{lcccc} 
Big problem & Medium problem & Small problem & Very small problem & No problem \\
0 & 1 & 2 & 3 & 4 \\
\hline
\end{tabular}

Overall satisfaction

11) Overall: during the past 30 days, how satisfied have you been with your sex life?

\begin{tabular}{lcccc} 
Very dissatisfied & Mostly dissatisfied & Neutral or mixed & Mostly satisfied & Very satisfied \\
0 & 1 & 2 & 3 & 4 \\
\hline
\end{tabular}


Appendix 2. Hospital Anxiety and Depression Scale

Tick the box beside the reply that is closest to how you have been feeling in the past week.

Don't take too long over you replies: your immediate is best.
D A
Questions
D A
Questions

I feel tense or 'wound up':

I feel as if I am slowed down:

\begin{tabular}{|lllll|}
\hline & 3 & Most of the time & 3 & Nearly all the time \\
\hline 2 & A lot of the time & 2 & Very often \\
\hline 1 & From time to time, occasionally & 1 & Sometimes \\
\hline & Not at all & 0 & Not at all \\
\hline & I still enjoy the things I used to enjoy: & & I get a sort of frightened feeling like 'butterflies' in the stomach: \\
\hline 1 & Definitely as much & Not quite so much & 0 & Not at all \\
\hline 2 & Only a little & 1 & Occasionally \\
\hline 3 & Hardly at all & 2 & Quite often \\
\hline
\end{tabular}

I get a sort of frightened feeling as if something awful is about to happen:

I have lost interest in my appearance:

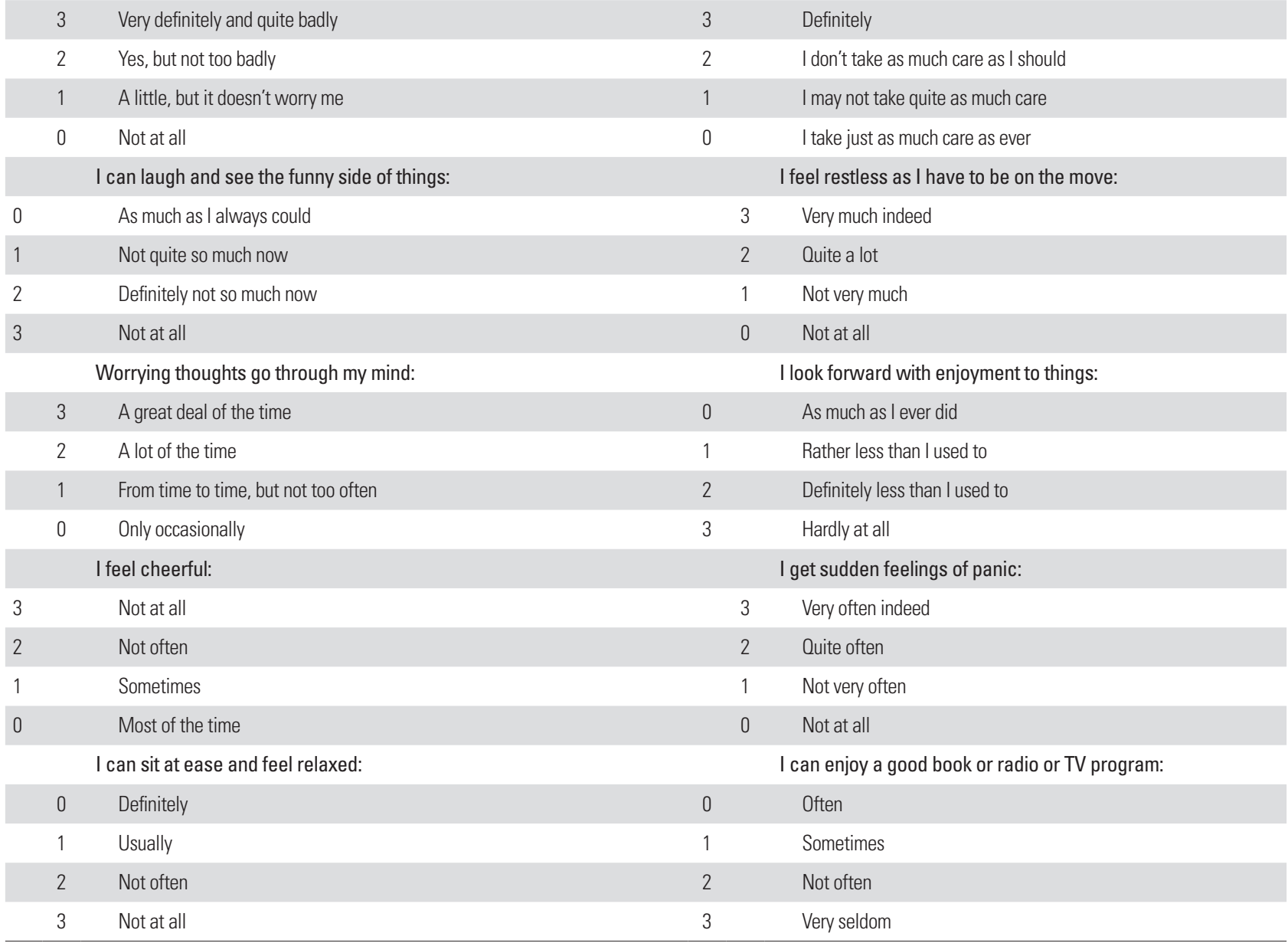

Please check you have answered all the questions.

\section{Scoring:}

Total score: depression (D)

Anxiety (A)

$0-7=$ normal

$8-10=$ borderline abnormal (borderline case)

11-21=abnormal (case) 